\title{
On the Rove Beetles (Coleoptera: Staphylinidae) from Danube Delta Biosphere Reserve in the Coleoptera Collections of "Grigore Antipa" National Museum of Natural History
}

\author{
Melania STAN* \\ This paper is dedicated to the memory of Dr. Stefan Negru and Dr. Medeea Weinberg \\ "Grigore Antipa” National Museum of Natural History, 1 Şos. Kiseleff, 011341 Bucharest 1, Romania \\ "corresponding author, e-mail: mstan@antipa.ro \\ Received: October 30, 2017; Accepted: November 24, 2017; Available online: December 28, 2017; Printed: December 31, 2017

\begin{abstract}
Grigore Antipa" National Museum of Natural History, collected from the Danube Delta Biosphere Reserve, between 1962-1966, in 1991, 1995 and 2012-2017. The material was collected mainly using light traps. Aleochara tenuicornis Kraatz, Carpelimus anthracinus (Mulsant \& Rey), Bledius frisius Lohse, Ochthephilum collare (Reitter) and Achenium propontiacum Bordoni are mentioned for the first time from this area.
\end{abstract}

Key words: Staphylinidae, Danube Delta Biosphere Reserve (Romania), Coleoptera Collections.

\section{INTRODUCTION}

The Danube Delta Biosphere Reserve (Romania) is an area of national and international importance; among its main ecosystems there are running waters, stagnant waters, wetlands and flooded areas, fluvial and marine levees, as well as human-modified areas (for agriculture, silviculture, pisciculture).

Since 1991, the Danube Delta Biosphere Reserve was listed as a wetland of international importance, especially as a waterflow habitat under the Ramsar Convention, and is among the largest of the 600 recognised wetlands. The universal natural heritage value of the reserve was recognised in 1991 by the inclusion of the strictly protected areas in the World Heritage List under the World Heritage Convention.

For this reserve, the rove beetle fauna is very poorly known. There are a few articles that deal with beetles from Letea Island and Caraorman Levee (Negru, 1968, Ieniștea, 1968, 1974), while other papers present a few rove beetle species collected from the Danube Delta Biosphere Reserve (Raianu, 1963; Stan 2004, 2010, 2012).

\section{MATERIALS AND METHODS}

Many rove beetle specimens, collected during 1962-1966 within the project The entomofauna of the Letea Island by the entomologists of "Grigore Antipa" National Museum of Natural History, were still unidentified. To these specimens, a material collected by the Museum researchers involved in the projects regarding the diversity of Romanian fauna $(1991,1995)$ and the diversity of insect fauna from Dobrudja $(2012,2015,2016)$ was added. The material was enriched by the donations made by Dr. Constantin Ciubuc in 2012 and 2017. Most specimens were collected using light traps. I studied rove beetle specimens which came from 23 collecting sites (Fig.1). 
The collecting sites from the Danube Delta Biosphere Reserve are: Chilia Veche (1), Letea Forest (Periprava, C. A. Rosetti), (2), Hașmacul Mare Natural Reserve (3), Letea (village) $-45.274^{\circ} \mathrm{N}, 29.526^{\circ} \mathrm{E}$, (4), Sulina (5), Litcov Channel (6), Maliuc, Mila 26 (7), Uzlina (Murighiol) (8), Caraorman Forest (Crișan, Caraorman), (9), Sfântu Gheorghe (10), Sacalin Island (11), Dunavăț (Murighiol) (12), Sarinasuf (Murighiol) (13), Popina

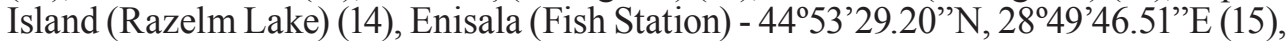
Jurilovca (16), Periteașca (17), Chituc Levee, Sinoe-Black Sea Channel - 44⒊'12.34”N, $28^{\circ} 53^{\prime} 57.82$ ”E (18), Nebunu Lake (Periprava) (19), Madgearu Channel (20) Murighiol

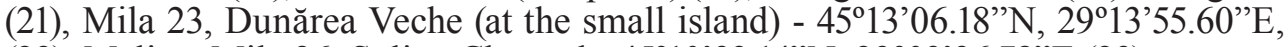

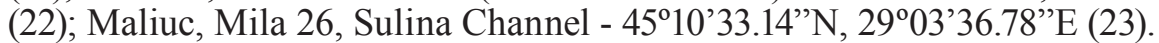

For morphological study a Stemi $2000-\mathrm{C}$ stereomicroscope was used. The photographs were taken using a Leica $205 \mathrm{C}$ stereomicroscope with a mounted Canon EOS 70 D camera. The specimens are preserved in the Coleoptera Collections of "Grigore Antipa" National Museum of Natural History, Bucharest, Romania.

Abbreviations for collector names:

A.P. - Angela Petrescu; A.P.-G.- Aurelian Popescu-Gorj; A.R. - Atena Roșca; C.A. - Costică Adam; C.C. - Cristina Constantinescu; C.Ci - Constantin Ciubuc; C.H. - Cristina Hoinic; D.R. - Dorel Ruşti; E.I. - Elena Iorgu; G.C. - Gabriel Chişamera; I.D. - Ion Drăghia; I.I. - Ionuț Iorgu; I.M. - Ioana Matache; I.S. - Igor Sienkiewicz; M.W. - Medeea Weinberg; N.G. - Nicolae Găldean; N.S. - Nicolae Săvulescu; R.S. - Rodica Serafim; Șt.N. - Ștefan Negru; X.S.-P. - Xenia Scobiola-Palade.

\section{RESULTS}

74 rove beetle species were identified examining the Museum collections. These species belong to 7 subfamilies: Tachyporinae (1), Aleocharinae (4), Oxytelinae (18), Oxyporinae (1), Steninae (2), Paedrinae (19) and Staphylininae (29). The number of the specimens, the collecting sites (alphabetically grouped), the collecting date, collecting method (where there is such information), and collector's name are mentioned for each species. For part of the collecting sites the geographical coordinates are indicated in the Material and Methods chapter, for other collecting sites the coordinates are mentioned below because there were different collecting dates for the same locality.

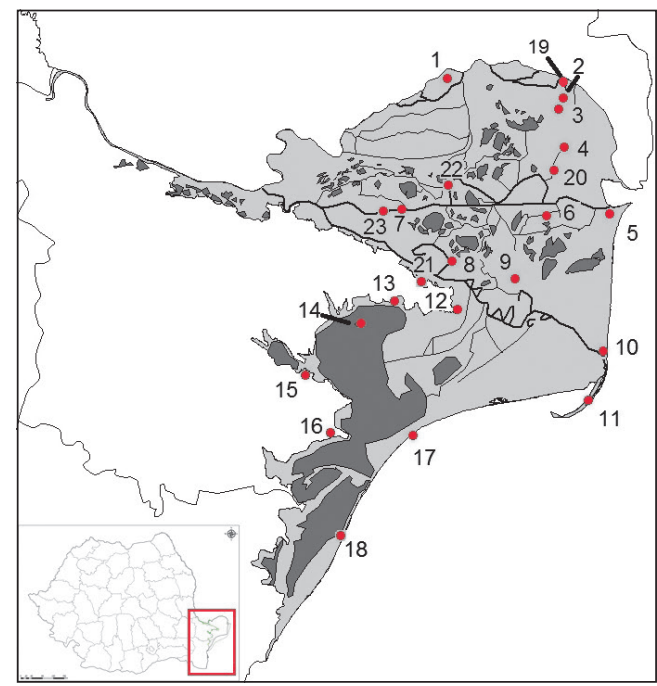

Fig. 1 - The map of collecting sites from the Danube Delta Biosphere Reserve 
Subfamily Tachyporinae

Tachyporus dispar (Paykull, 1789)

Examined material: 1 ( (immature), 1 +, Maliuc, Mila 26, Sulina Channel, 26.06.2002, mercury vapor light trap, leg. C.Ci.

Subfamily Aleocharinae

Aleochara curtula (Goeze, 1777)

Examined material: 1 , , Sarinasuf $\left(44.9964^{\circ} \mathrm{N}, 29.1017^{\circ} \mathrm{E}\right), 15.08 .2015$, leg. I.I.

Aleochara tenuicornis Kraatz, 1856 (Fig. 2 A)

Examined material: 12 गे, 7 क , Chituc Levee, Sinoe-Black Sea Channel, 17.08.2003, black light trap, leg. C.Ci.; 3 , 3 क , Enisala, 14.08.2003, black light trap, leg. C.Ci.; 11 ô, 5 \%, Sfântu Gheorghe, 12.06.1995, leg. D.R.

Dacrila fallax (Kraatz, 1856)

Examined material: 1 q, Mila 23, 31.07.2002, white light trap, leg. C.Ci.

Nehemitropia lividipennis (Mannerheim, 1830)

Examined material: 1 9, Maliuc, Mila 26, 6-9.08.1991, light trap, leg. I.M.\&R.S.; 1 §, Maliuc, Mila 26, Sulina Channel, 26.06.2002, mercury vapor light trap, leg. C.Ci.

Subfamily Oxytelinae

Anotylus rugosus (Fabricius, 1775)

Examined material: Caraorman Forest: 1 ô, 23.06.1963, leg. N.S.; 4 specs, 20.06.1967, leg. Șt.N.; 2 specs, 8.10.1968, leg. Șt.N.; 2 specs, 21.07.1979, leg. A.P.-G.; 1 spec., Dunavăt, 31.05.1994, leg. C.H.; Enisala: 1 + 14.08.2003, black light trap, leg. C.Ci.; 1 + 16.08.2003, mercury vapor light trap, leg. C.Ci.; Letea Forest: 3 specs, 23.06.1966, leg. S.t.N.; Letea (village): 1 spec., on willow bark covered with moss, 4.05.2012, leg. C.C.; 1 Oे, 2 क , 30.06.2016, black light trap, leg. I.I.; 48 specs, Maliuc, Mila 26, light trap, 6-9.08.1991, leg. I.M.\&R.S.; 17 specs, Maliuc, Mila 26, Sulina Channel, 26.06.2002, mercury vapor light trap, leg. C.Ci.; 1 ภ, 1 O, Mila 23, 31.07.2002, white light trap, leg. C.Ci.; 1 ô, Murighiol, 3.08.1979, leg. ?; 5 क, Nebunu Lake, 7.07.2015, leg. E.I.; Periprava: 1 spec., 30.04.1965, leg. Șt.N.; 1 spec., 24.06.1965, leg. Șt.N.; 2 specs, 14.10.1966, leg. Șt.N.; 1 o , 7.07.2015, leg. E.I.; 1 spec., Popina Island, 6.09.1988, leg.?

Bledius diota Schiödte, 1866

Examined material: 2 ô, 1 क, Enisala, 14.08.2003, black light trap, leg. C.Ci.; 1 ภ, 2 , Chituc Levee, Sinoe-Black Sea Channel, 17.08.2003, black light trap, leg. C.Ci.; 1 \&, Sulina, 8.07.1968, leg. M.W.

Bledius dissimilis Erichson, 1840

Examined material: 11 specs, Crișan, 24.06.1967, leg. Șt.N. \& M.W.

Bledius frisius Lohse, 1978 (Fig. 2 B a, b)

Examined material: Caraorman Forest: 4 ô, 20 , , 20.06.1967, leg. St.N.; 4 q , 21.06.1967, leg. Șt.N.; 1 + 22.09 .1967$, leg. Șt.N.; 40 Oे, 186 , 24.09 .1967 (light traps), leg. Șt.N.; 1 ô, 5 q, 02.06.1969, leg. M.W.; 11 specs, 29.08.1969, leg. Șt.N.; 4 + , 30.09.1969, leg. Șt.N.; C. A. Rosetti: 2 Ò, 2 q, 20.07.1964, leg. Șt.N.; 1 \%, 5.07.1966, leg. Șt.N.; 100 ô, 196 + , Chituc Levee, Sinoe-Black Sea Channel, 17.08.2003, black light traps, leg. 

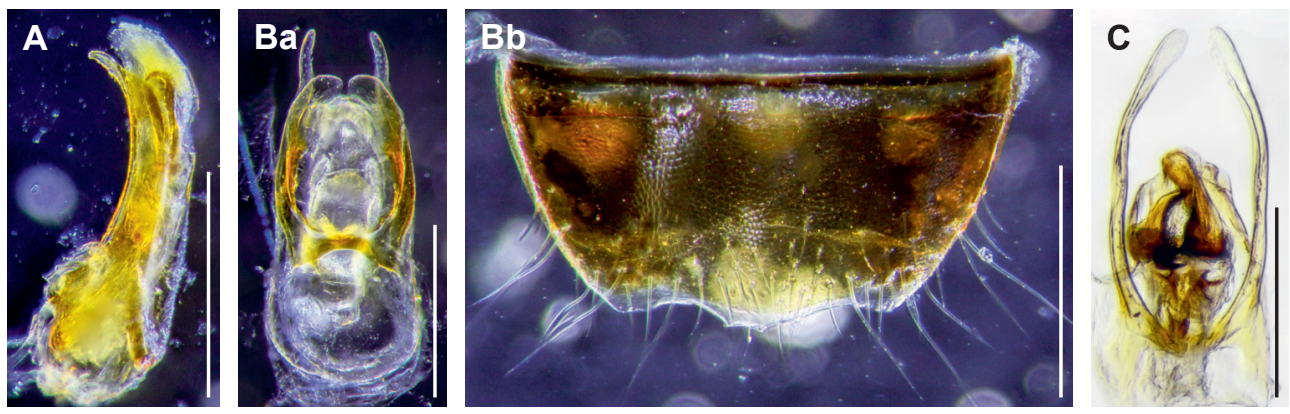

Fig. 2 - A: Aleochara tenuicornis Kraatz: median lobe (lateral view); B: Bledius frisius (Lohse): a aedeagus (ventral view), b - male sternite VIII; C: Carpelimus anthracinus (Mulsant \& Rey): aedeagus (ventral view). Scale bars: A, B $-0.5 \mathrm{~mm}, \mathrm{C}-0.25 \mathrm{~mm}$.

C.Ci.; 11 specs, Crișan, 14.07.1970, leg. M.W.; Enisala: 1 đ̊, 3 , 14.08.2003, black light trap, leg. C.Ci.; 3 गे, 2 ㅇ, 15.08.2003, white light trap, leg. C.Ci.; 1 spec., Letea Forest; 12.07.1980, leg. A. P.-G.; Letea (village): 2 中, 4.05.2012, on willow bark covered with moss, leg. C.C.; 7 , 30.06.2016, black light trap, leg. I.I.; 3 specs, Maliuc, Mila 26, 6.08.1991, light trap, leg. I.M.; 1 ō, Mila 23, 31.07.2002, white light trap, leg. C.Ci; 7 ๆ, Nebunu Lake, 7.07.2015, leg. E.I.; Periprava: 10 ๆ, 30.04.1965, leg. Șt.N.; 6 ô, 21 †, 1.07.1965, leg. Șt.N.; 1 ○, Periteaşca, 17.06.1995, leg. D.R.; 6 specs, Popina Island, 6.09.1988, leg. ?; Sfântu Gheorghe: 3 q, 14.08.1971, leg. X.S.-P.; 5 Õ, 15 q, 12.06.1995, leg. D.R.; Sulina: 1 q, 18.06.1964, leg. Șt.N.; 2 ò, 9 †, 23.07.1964, leg. Șt.N.; 3 ๆ, 17.06.1965, leg. Șt.N.; 3 o, 20-23.07.1966, leg. Șt.N.; 1 ô, 1 ㅇ, 12.07.1967, leg. A.R.;

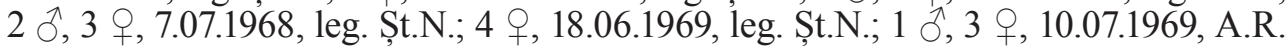

\section{Bledius furcatus (Olivier, 1811)}

Examined material: 4 ふ̋, 2 †, Chituc Levee, Sinoe-Black Sea Channel, 17.08.2003, black light trap, leg. C.Ci., leg. C.Ci.; 4 ô, 4 ㅇ, Sfântu Gheorghe, 12.06.1995, leg. D.R.

\section{Bledius gallicus (Gravenhorst, 1806)}

Examined material: Caraorman Forest: 45 specs, 20.06.1967, leg. Șt.N.; 10 specs, 21.06.1967, leg. Șt.N.; 6 specs, 23.06.1967, leg. Șt.N.; 12 specs, 24.06.1967, leg. Șt.N. \& M. W.; 1 spec., 31.05.1968, leg. I.D.; 2 specs, 5.07.1968, leg. A.P.-G.; 5 specs, 30.08.1969, leg. Șt.N.; 12 specs, 21.09.1969, leg. Șt.N.; 4 ō, 5 o, 21.09.1969, leg. Șt.N.; 16 specs, 30.09.1969, leg. Șt.N.; Crișan: 1 spec., 25.08.1969, leg. Șt.N.; 3 specs, 14.07.1970, leg. M.W.; 1 spec., 15.07.1970, leg. Șt.N.; Enisala: 1 ò, 6 q, 14.08.2003, black light trap, leg. C.Ci.; $1 \hat{\jmath}$, 16.08.2003, mercury vapor light trap, leg. C.Ci.; Letea Forest: 1 spec., 3.07.1963, leg. A.P.-G.; 1 spec., 23.06.1966, leg. Șt.N.; 1 đ, 2 q, Letea (village), 30.06.2016, black light trap, leg. I.I.; 2 đ̃, 15 O, Maliuc, Mila 26, 6-9.08.1991, light trap, leg. I.M.\&R.S.; 10 , Maliuc, Mila 26, Sulina Channel, 26.06.2002, mercury vapor light trap, leg. C.Ci.; 28 specs, Mila 23, 31.07.2002, white light trap, leg. C.Ci; 3 गे, 3 क्, Nebunu Lake, 7.07.2015, leg. E.I.; 5 specs, Periprava, 24.06.1966, leg. Șt.N.; Sulina: 1 ふै, 12-14.07.1966, A.P.-G.; 1 spec., 08.07.1968, M.W.

Bledius tricornis (Herbst, 1784)

Examined material: C. A. Rosetti: 1 spec., 21.07.1964, leg. A.P.-G.; 2 specs, 05.07.1965, leg. Șt.N.; Caraorman Forest: 3 ô, 23.06.1963, leg. N.S.; 2 specs, 18.06.1967, leg. Șt.N.; 
7 specs, 20.06.1967, leg. Șt.N., 15 specs, 21.06.1967, leg. Șt.N.; 3 specs, 24.09.1967, leg. Șt.N.; 1 spec., 31.05.1968, leg. I.D.; 9 specs, 2.06.1968, leg. M.W.; 1 spec., 03.07.1968, leg. A.P.-G.; 11 specs, 5.07.1968, A.P.-G.; 2 specs, 6.07.1968, leg. M.W.; 1 spec., 29.08.1969, leg. Șt.N.; 3 q, 21.09.1969, leg. Șt.N.; Caraorman (village): 2 specs, 23.06.1967, leg. Șt.N.; 27 specs, 21.07.1979, leg. A.P.-G.; 1 spec., 22.07.1979, leg. A.P.-G.; 1 +, Chituc Levee, Sinoe-Black Sea Channel, 17.08.2003, black light traps, leg. C.Ci.; Crișan: 4 specs, 17.08.1967, leg. M.W.; 1 spec., 25.08.1969, leg. Șt.N., 3 specs, 14.07.1970, leg. M.W.; Enisala: 2 O, 9 ㅇ, 14.08.2003, black light trap, leg. C.Ci.; 5 + 16.08 .2003 , mercury vapor light trap, leg. C.Ci.; 2 $\hat{\text {, }}, 17$ + , Letea (village), 30.06.2016, black light trap, leg. I.I.; 4 क, Madgearu Channel, 20-21.06.1993, leg. C.H.; 3 ô, 1 क, Maliuc, Mila 26, 6-9.08.1991, leg. I.M.\&R.S.; 1 §ै, 4 ㅇ, Maliuc, Mila 26, Sulina Channel, 26.06.2002, mercury vapor light trap, leg. C.Ci.; 1 స, 18 क , Mila 23, 31.07.2002, white light trap, leg. C.Ci; 9 ô, 17 o, Murighiol, 3.08.1979, leg. ?; 10 ô, 37 ㅇ, Nebunu Lake, 7.07.2015, leg. E.I.; Periprava: 10 specs, 30.04.1965, leg. Șt.N., 2 specs, 4.07.1965, leg. Șt.N.; 5 specs, 14.07.1966, leg. X.S.-P.; 1 spec., 14.10.1966, leg. Șt.N.; 1 spec., 24.06.1966, leg. Șt.N.; 1 q, 7.07.2015, leg. E.I.; 1 spec., Popina Island, 6.09.1988, leg. ?; Sfântu Gheorghe: 1 spec., 14.08.1971, leg. X.S.; 4 specs, 7.09.1971; Sulina: 9 specs, 23.07.1964, leg. Șt.N.; 9 specs, 20-23.07.1966, leg. Șt.N., 4 specs, 9.07.1967, leg. Șt.N.; 1 spec., 7.07.1968, leg. Șt.N., 10 spec., 8.07.1968, leg. Șt.N.\& M.W.; 4 specs, 9-12.07.1969, leg. A.R.

Bledius unicornis (Germar, 1825)

Examined material: 1 ô, C. A. Rosetti, 20.07.1964, leg. Șt.N.; 4 o, Letea (village), 30.06.2016, black light trap, leg. I.I.; 2 ô, 2 \%, Mila 23, 31.07.2002, white light trap, leg. C.Ci.

\section{Bledius verres Erichson, 1840}

Examined material: 1 ㅇ, Caraorman Forest, 21.09.1969, leg. Șt.N.; 3 specs, Crișan, 17.08.1967, leg. M.W.; 1 spec., Periprava, 29.07.1963, leg. A.P.-G.; 1 spec., Sacalin Island, 5.07.1971; 1 spec., Sfântu Gheorghe, 12.06.1995, leg. D.R.

\section{Carpelimus anthracinus anthracinus (Mulsant \& Rey, 1861) (Fig 2 C)}

Examined material: 1 ڤึ, Chituc Levee, Sinoe-Black Sea Channel, 17.08.2003, black light trap, leg. C.Ci.; 14 specs, Enisala, 16.08.2003, mercury vapor light trap, leg. C.Ci.; 8 O, 11 o, Letea (village), 30.06.2016, black light trap, leg. I.I.; 4 ô, 9 o, Mila 23, 31.07.2002, white light trap, leg. C.Ci; 2 +, Sfântu Gheorghe, 12.06.1995, leg. D.R.

\section{Carpelimus corticinus (Gravenhorst, 1806)}

Examined material: 1 $\widehat{\text { Tे }}$, Enisala, 16.08.2003, mercury vapor light trap, leg. C.Ci.; 1 §, 2 ㅇ, Mila 23, 31.07.2002, white light trap, leg. C.Ci.

Carpelimus exiguus (Erichson, 1839)

Examined material: 4 ô, 2 9 , Enisala, 16.08.2003, mercury vapor light trap, leg. C.Ci.; 4 , , Mila 23, 31.07.2002, white light trap, leg. C.Ci.

Carpelimus gracilis (Mannerheim, 1830)

Examined material: 1 ก๊, Enisala, 14.08.2003, black light trap, leg. C.Ci. 
Carpelimus impressus (Lacordaire, 1835)

Examined material: 1 ○े, Enisala, 16.08.2003, mercury vapor light trap, leg. C.Ci.

Carpelimus nitidus (Baudi di Selve, 1848)

Examined material: 1 ô, Letea (village), 30.06.2016, black light trap, leg. I.I.

Carpelimus politus politus (Kiesenwetter, 1850)

Examined material: 3 Ĵ, 2 9, Chituc Levee, Sinoe-Black Sea Channel, 17.08.2003, black light traps, leg. C.Ci.; 7 specs, Enisala, 16.08.2003, mercury vapor light trap, leg. C.Ci.; 4 specs, Mila 23, 31.07.2002, white light trap, leg. C.Ci.

Oxytelus piceus (Linnaeus, 1767)

Examined material: 1 \&, Enisala, 16.08.2003, mercury vapor light trap, leg. C.Ci.; 4 ô, Maliuc, Mila 26, 6-9.08.1991, leg. I.M.\&R.S.; Maliuc, Mila 26, Sulina Channel, $4 \hat{\partial}, 26.06 .2002$, mercury vapor light trap, leg. C.Ci.; Periprava: $1 \stackrel{\lambda}{\partial}, 14.09 .1966$, leg. Șt.N.; 2 specs, 12.10.1966, leg. Șt.N.

Planeustomus heydeni heydeni (Eppelsheim, 1884)

Examined material: 1 Oे, Caraorman, 21.06.1967, leg. Șt.N.; 1 त, 13.06.1969, leg. Șt.N.; 2 specs., C. A. Rosetti, 5.07.1965, leg. Șt.N.; 3 specs, Mila 23, 31.07.2002, white light trap, leg. C.Ci.

\section{Subfamily Oxyporinae \\ Oxyporus rufus (Linnaeus, 1758)}

Examined material: 6 specs, Letea Forest, 24.06.1958, leg. ?

Subfamily Steninae

Stenus clavicornis (Scopoli, 1763)

Examined material: 1 spec., Periprava, 14.10.1966, leg. Șt. N.

Stenus comma (Le Conte, 1863)

Examined material: 2 specs, Crișan, 18.07.1970, leg. Șt. N.

Subfamily Paederinae

Achenium humile (Nicolai, 1822) (Fig. 3 A)

Examined material: 2 \%, Mila 23, 31.07.2002, white light trap, leg. C.Ci.

Achenium propontiacum Bordoni, 2009 (Fig. 3 B a, b)

Examined material: 2 ภ, 3 9 , Maliuc, Mila 26, light trap, 6-9.08.1991, leg. I.M.\&R.S.; 2 , Mila 23, 31.07.2002, white light trap, leg. C.Ci.

\section{Lathrobium elongatum (Linnaeus, 1767)}

Examined material: 2 ㅇ, Enisala, 14.08.2003, black light trap, leg. C.Ci.; Letea (village): 1 ภ, 2 q, on willow bark covered with moss, 4.05.2012, leg. C.C.; 3 $\lambda, 15$ †, 30.06.2016, black light trap, leg. I.I.; 1 , Madgearu Channel, 20-21.06.1993, leg. C.H.; 2 O, 8 o, Maliuc, Mila 26, 6-9.08.1991, light trap, leg. I.M.\&R.S.; 1 ô, 7 , Maliuc, Mila 26, Sulina Channel, 26.06.2002, mercury vapor light trap, leg. C.Ci.; 1 ô, 1 q, Mila 23, 31.07.2002 white light trap, leg. C.Ci.; 2 q, Periprava, 7.07.2015, leg. E.I.; 1 Oे, Sfântu Gheorghe, 12.06.1995, leg. D.R. 

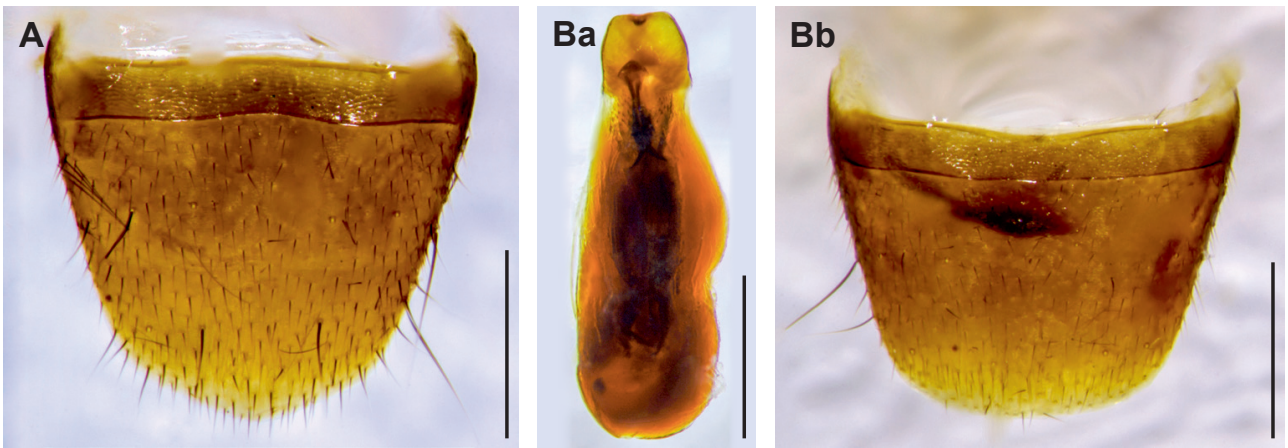

Fig. 3 - A: Achenium humile (Nicolai): female sternite VIII; B: Achenium propontiacum Bordoni: $\mathrm{a}$ - aedeagus (dorsal view), b - female sternite VIII. Scale bars: $0.5 \mathrm{~mm}$.

Lathrobium fulvipenne (Gravenhorst, 1806)

Examined material: 1 万ै, Maliuc, Mila 26, Sulina Channel, 26.06.2002, mercury vapor light trap, leg. C.Ci; 1 O, Mila 23, 31.07.2002, white light trap, leg. C.Ci.

\section{Lathrobium furcatum Czwalina, 1888}

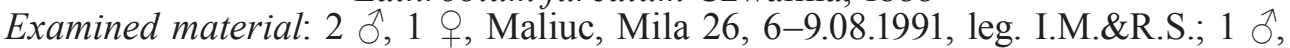
Periprava, 7.07.2015, leg. E.I.

Lathrobium impressum Heer, 1841

Examined material: 1 đे, Letea Forest, rotten reeds, 4.05.2012, leg. C.C.

Lathrobium taxi (Bernhauer, 1902)

Examined material: 1 đ̊, 3 क, Maliuc, Mila 26, 6-9.08.1991, leg. I.M.\&R.S.

Leptobium gracile (Gravenhorst, 1802)

Examined material: 1 ô, 3 q, Mila 23, 31.07.2002, white light trap, leg. C.Ci.

Lithocharis nigriceps Kraatz, 1859

Examined material: 1 §ै, 1 \%, Enisala, 14.08.2003, black light trap, leg. C.Ci.

Ochthephilum collare (Reitter, 1884)

Examined material: 1 9, Caraorman, 4.05.1968, leg. Șt.N.; 6 o, Enisala, 16.08.2003, mercury vapor light trap, leg. C.Ci.; 5 , Letea (village), 30.06.2016, black light trap, leg. I.I.; 2 O, 3 9, Maliuc, Mila 26, 6-9.08.1991, leg. I.M.\&R.S.; 8 ㅇ, Maliuc, Mila 26, Sulina Channel, 26.06.2002, mercury vapor light trap, leg. C.Ci.; 2 , Mila 23, 31.07.2002, white light trap, leg. C.Ci.; $1 \curvearrowright$, Nebunu Lake, 7.07.2015, leg. E.I.

\section{Paederus balcanicus Koch, 1938}

Examined material: Caraorman Forest: 1 spec., 11.05.1967, leg. X.S-P.; 1 spec., 25.09.1967, leg. Șt.N.; 2 +, 13.05.1968, leg. X.S.-P.; 3 specs, 5.10.1968, leg. Șt.N.; 1 spec., 15.06.1969, leg. Șt.N.; C.A. Rosetti: 13 specs, 5.07.1965, leg. Șt.N.; 3 specs, 5.07.1966, leg. St.N.; 6 specs, Letea Forest, 3.04.2009, leg. I.I.; Letea (village): 12 specs,

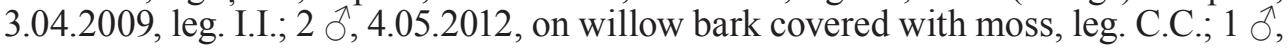
30.06.2016, black light trap, leg. I.I.; 19 specs, Maliuc, Mila 26, 6.08.1991, leg. R.S.; 
1 đ̊, 9.08.1991, leg. R.S.; 2 ㅇ, Maliuc, Mila 26, Sulina Channel, 26.06.2002, mercury vapor light trap, leg. C.Ci.; 12 specs, Mila 23, 31.07.2002, white light trap, leg. C.Ci.; 2 ${ }^{7}, 3$ q, Nebunu Lake, 7.07.2015, leg. E.I.; Periprava: 1 spec., 18.07.1964, leg. Șt.N.; 1 spec., 1.07.1965, leg. Șt.N.; 1 spec., 16.07.1966, leg. X.S.-P.; 2 specs, 8.05.1969, leg. M.W.; 1 spec., Periteașca Lake (Periteașca-Leahova complex), 17.06.1995, leg. D.R.; Sfântu Gheorghe: 1 spec., 14.08.1971, leg. X.S.-P.; 1 P, 9.07.1972, leg. A.P.-G.; 4 ô, 9 + , 12.06.1995, leg. D.R.; Sulina: 1 ô, 18.06.1964, leg. Șt.N.; 1 spec., 23.07.1964, leg. Șt.N.; 2 specs, 9.07.1967, leg. Șt.N.; 2 specs, 7.07.1968, leg. Șt.N.; 1 spec., 10.07.1969, leg. A.R.; 1 ơ, Uzlina (chalet), 18.06.1991, leg. N.G.

\section{Paederus fuscipes Curtis, 1826}

Examined material: Caraorman Forest: 18 specs, 6-10.05.1967, leg. M.W.\&X.S.-P.: 2 specs, 12.05.1967, leg. M.W.; 1 spec., 17.05.1967, leg. X.S.-P.; 20 specs, 23.09.1967, under cow dung, leg. Șt.N.; 2 specs, 25.09.1967, leg. M.W.; 1 spec., 12.05.1968, leg. X.S.-P.; 2 specs, 4.10.1968, leg. Șt.N.; 12 specs, 5.10.1968, leg. X.S.-P.; 1 spec., 8.10.1968, leg. X.S.-P.; 1 spec., 15.06.1969, leg. Șt.N.; 2 specs, 21.09.1969, leg. M.W.; 1 spec., 05.10.1970, on bark of Alnus glutinosa, leg. Șt.N.; 1 spec., 24.08.1992, leg. A.P.; C.A. Rosetti: 1 spec., 5.07.1966, leg. Șt.N.; 2 specs, 10.05.1967, Șt.N.; Chilia Veche: 4 specs, 7.05.1964, leg. ?; 12 specs, Chituc Levee, Sinoe-Black Sea Channel, 17.08.2003, black light traps, leg. C.Ci.; Crișan: 2 specs, 18.06.1967, leg. Șt.N.; 3 specs, 26.09.1967; leg. Șt.N.; 6 specs, 27.09.1967, leg. Șt.N.; 2 specs, 1.07.1968, leg.Șt. N.; 9 specs, 18.07.1970, leg. Șt.N.; 1 spec., 21.07.1970, leg. Șt.N.; Dunavățu de Jos: 1 spec., 31.05.1994, leg. C.H., 2 specs, 9.06.1994, leg. C.H.; Enisala: 1 + , 14.08.2003, black light trap, leg. C.Ci.; 1 ㅇ, 16.03.2003, mercury vapor light trap, leg. C.Ci.; 1 , Grindul Lupilor Reserve, 15.06.1995, leg. D.R.; Letea Forest: 3 specs, 9.05.1963, leg. ?; 2 specs, 11.05.1964, leg. X.S.-P.; 10 specs, 8.05.1964, leg. M.W.; 1 spec., 12.05.1964, leg. X.S.-P.; 3 specs, 13.05.1964, leg. X. S.-P; 1 spec., 26.06.1966, leg. Șt.N.; Letea (village): 10 specs, 3.04.2009, leg. I.I.; 2 ô, 2 + , 4.05.2012, leg. I.I.; 6 specs, Maliuc, Mila 26, 7.08.1991, leg. I.M.; 1 o, 1 क, Maliuc, Mila 26, Sulina Channel, 26.06.2002, mercury vapor light trap, leg. C.Ci.; 3 specs, Mila 23, 31.07.2002, white light trap, leg.

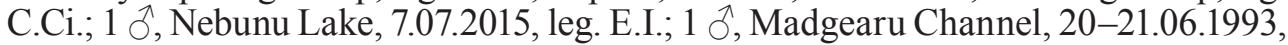
leg. C.H.; Periprava: 3 specs, 5.10.1963, leg. X.S.-P.; 1 spec., 9.05.1964, leg. X.S.-P.; 1 spec., 30.05.1965, leg. Șt.N.; 2 specs, 17.07.1966, leg. Șt.N.; 6 specs, 11.10.1966, leg. Șt.N.; 3 specs, 10.10.1966, under logs of Populus alba, leg. Șt.N.; 1 9, 7.07.2015, leg. E.I.; Sfântu Gheorghe: 1 spec., 22.10.1970, leg. Șt.N.; 1 spec., 14.08.1971, leg. X.S.-P.; 1 o, 12.06.1995, leg. D.R.; 1 spec., 27.05.1994, leg. C.H.; Sulina: 1 spec., 10.09.1966, leg. X.S.-P.; 1 spec., 9.07.1967, leg. Șt.N.; 1 spec., 12.07.1969, leg. A.R.

Paederus littoralis Gravenhorst, 1802

Examined material: 1 spec., Periprava, 11.10.1966, leg. Şt.N.

Paederus riparius (Linnaeus, 1758)

Examined material: Caraorman Forest: 1 spec., 11.08.1967, leg. X.S.-P.; 2 q, 21.09.1967, under dry cow dung, leg. Șt.N.; 1 spec., 25.09.1967, leg. M.W.; 4 specs, 10.06.1969, on the soil, leg. Șt.N.; 1 spec., 14.06.1969, leg. Șt.N.; 1 o, 15.06.1969, leg. Șt.N.; Chilia Veche: 1 spec., 15.04.1966, leg. Șt.N.; Crișan: 4 specs, 18.06.1967, leg. Șt.N.; 1 spec., 12.07.1970, leg. Șt.N.; 2 specs, 18.07.1970, leg. Șt.N.; 2 specs, Dunavățu de Jos, 31.05.1994, leg. C.H.; Letea Forest: 1 spec., 28.07.1963, leg. A.P.-G.; 1 spec., 10.05.1964; 2 specs, Litcov Channel, 1.09.1969, leg. Șt.N.; 6 specs, Maliuc, Mila 26, 
6.08.1991, leg. R.S.; 5 Oૈ, 8 o, 7.08.1991, leg. I.M.; 6 specs, Mila 23, 31.07.2002, white light trap, leg. C.Ci.; Periprava: 4 specs, 4.07.1965, leg. Șt.N.; 1 q, 16.07.1966, leg. Șt.N.; 1 + 7.07.2015, leg. E.I.; C.A. Rosetti: 6 specs, 5.07.1965, leg. Șt.N.; Sulina: 1 spec., 24.08.1964, leg. Șt.N.; 2 specs, 9.07.1967, leg. Șt.N.; 1 spec., 10.07.1969, leg. A.R.; Sfântu Gheorghe: 1 spec., 25.05.1971; 2 specs, 12.06.1995, leg. D.R.

Paederus schoenherri (Czwalina, 1889)

Examined material: 1 ô, Caraorman Forest, 15.06.1969, leg. Șt.N.

Pseudomedon obsoletus (Nordmann, 1837)

Examined material: 1 , , Chituc Levee, Sinoe-Black Sea Channel, 17.08.2003, black light trap, leg. C.Ci.; Enisala: 2 o, 14.08.2003, black light trap, leg. C.Ci.; 2 Oे, 3 q, 16.08.2003, mercury vapor light trap, leg. C.Ci.; Letea (village): $1 \hat{\jmath}, 4.05 .2012$, on willow bark covered with moss, leg. C.C.; 2 +, 30.06.2016, black light trap, leg. I.I.; 2

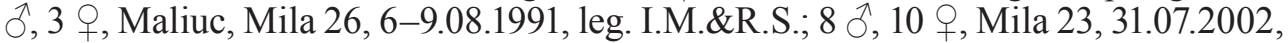
white light trap, leg. C.Ci; 1 §, 2 , Uzlina (chalet), 18.06.1991, leg. N.G.

Scopaeus debilis Hochhuth, 1851

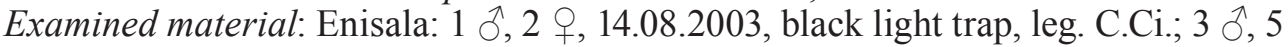
+, 16.08.2003, mercury vapor light trap, leg. C.Ci.; 2 +, Maliuc, Mila 26, 6-9.08.1991, leg. I.M.\&R.S.

Scopaeus laevigatus (Gyllenhal, 1827)

Examined material: 1 o, Chituc Levee, Sinoe-Black Sea Channel, 17.08.2003, black

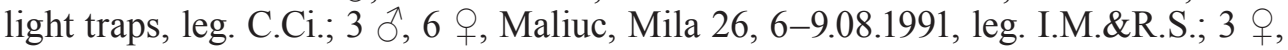
Mila 23, 31.07.2002, white light trap, leg. C.Ci.

Tetartopeus rufonitidus (Reitter, 1909)

Examined material: 5 đo, 9 q, Enisala, 14.08.2003, black light trap, leg. C.Ci.; 3 q, Letea

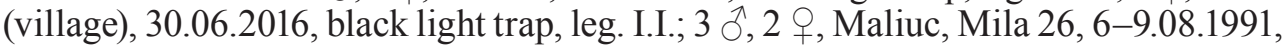
leg. I.M.\&R.S.; 1 , , Maliuc, Mila 26, Sulina Channel, 26.06.2002, mercury vapor light trap, leg. C.Ci.; 1 O, Mila 23, 31.07.2002, white light trap, leg. C.Ci.; 1 Ò, Sfântu Gheorghe, 12.06.1995, leg. D.R.

Subfamily Staphylininae Atanygnathus terminalis (Erichson, 1839)

Examined material: 1 ऽ, Mila 23, 31.07.2002, white light trap, leg. C.Ci.

Cafius xantholoma (Gravenhorst, 1806)

Examined material: 1 ô, 2 9, Chituc Levee, Sinoe-Black Sea Channel, 17.08.2003, black light traps, leg. C.Ci.; 1 \&, Sfântu Gheorghe, 12.06.1995, leg. D.R.

Creophilus maxillosus (Linnaeus, 1758)

Examined material: 1 §ै, 3 9 , Chilia Veche, 15.04.1966, leg. Șt. N.; 1 spec., Maliuc, Mila 26, 6.08.1991, leg. R.S.

Dinothenarus pubescens (De Geer, 1774)

Examined material: 1 spec., Periprava, 23.06.1963, leg. N.S. 
Gabrius femoralis (Hochhuth,1851)

Examined material: 2 ô, Enisala, 29.06.1956, leg.?

Gabrius osseticus (Kolenati, 1846)

Examined material: 1 q, Periprava, 7.07.2015, leg. E.I.

Gabrius suffragani Joy, 1913

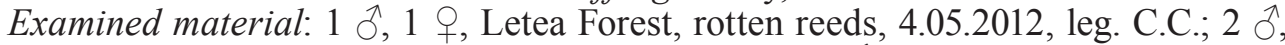
2 ㅇ, Maliuc, Mila 26, 6-9.08.1991, leg. I.M.\&R.S.; 1 Ĵ, 1 , Mila 23, 31.07.2002, white light trap, leg. C.Ci.

\section{Leptacinus sulcifrons (Satephens, 1833)}

Examined material: 1 ō, 2 q, Letea (village), 30.06.2016, black light trap, leg. I.I.

Neobisnius procerulus procerulus (Gravenhorst, 1806)

Examined material: 1 q, Maliuc, Mila 26, 6-9.08.1991, leg. I.M.\&R.S.; 1 q, Mila 23, 31.07.2002, white light trap, leg. C.Ci.

\section{Ocypus nitens (Schrank, 1781)}

Examined material: Periprava: 1 spec., 5.11.1964, leg. N.S.; 1 spec., 17.04.1966, leg. Şt.N.; 1 spec., 17.10.1974, leg. N.S.

Ocypus ophthalmicus ophthalmicus (Scopoli, 1763)

Examined material: 1 đ̃, Hașmacul Mare Natural Reserve, 10.06.1995, leg. I.M.

Ocypus picipennis picipennis (Fabricius, 1793)

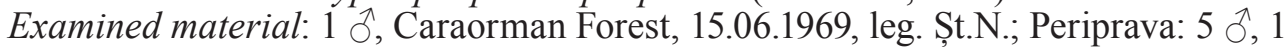
†, 1.04.1966, leg. Șt.N.

Philonthus caucasicus Nordmann, 1837

Examined material: 2 ô, 1 †, Periprava (village), 15.10.1966, leg. Șt.N.

Philonthus concinnus (Gravenhorst, 1802)

Examined material: Caraorman Forest: 1 गे, 22.09.1967, leg. Șt.N.; 1 ô, 15.06.1969, leg. Șt.N.; 1 गे, Maliuc, Mila 26, 6-9.08.1991, leg. I.M.\&R.S.; 3 + , Periprava (village), 1.04.1966, leg. Şt.N.

Philonthus dimidiatipennis Erichson, 1840

Examined material: $1 \stackrel{\jmath}{\jmath}$, Chituc Levee, Sinoe-Black Sea Channel, 17.08.2003, black light trap, leg. C.Ci.; 1 , Enisala, 14.08.2003, black light trap, leg. C.Ci.; 2 , , Mila 23, 31.07.2002, white light trap, leg. C.Ci.

\section{Philonthus fumarius (Gravenhorst, 1806)}

Examined material: 1 spec., Crișan, 10.10.1968, leg. X.S.-P.

Philonthus micans (Gravenhorst, 1802)

Examined material: 1 đ̃, 1 q, Crișan, 13.07.1970, leg. Șt.N.; Letea (village): 6 đ, 7 q, 30.06.2016, black light trap, leg. I.I.; 1 spec., 4.05.2012, rotten reeds, leg. C.C.; 19 ô, 8 ㅇ, Maliuc, Mila 26, 6-9.08.1991, light trap, leg. I.M.\&R.S.; 2 ơ, Maliuc, Mila 26, 
Sulina Channel, 26.06.2002, mercury vapor light trap, leg. C.Ci.; 3 ๙ै, 1 , Mila 23, 31.07.2002, white light trap, leg. C.Ci.; 1 O, 3 \%, Nebunu Lake, 7.07.2015, leg. E.I.; Periprava (village): 1 क , 4.07.1965, leg. Șt.N.; 1 ${ }^{\lambda}, 14.10 .1966$, leg. X.S.-P.

\section{Philonthus politus (Linnaeus, 1758) \\ Examined material: 1 9, C.A. Rosetti, 20.07.1964, leg. X.S.-P.}

\section{Philonthus punctus (Gravenhorst, 1802)}

Examined material: C.A. Rosetti: 4 specs, 5.07.1965, leg. Șt.N.; 2 specs, 5.07.1966, leg. Șt.N.; Caraorman Forest: 1 ō, 18.06.1967, leg. Șt.N.; 1 क̊, 16.05.1968, leg. XS.-P.; 1 spec., 12.05.1968, leg., X.S.-P.; 1 spec., 6.06.1968, leg. I.D.; 1 spec., 6.07.1968, leg. M.W.; 2 đ̃, 5.07.1968, leg. Șt.N.; 2 spec., 12.06.1969, leg. M.W.; 1 spec., 29.08.1969, leg. Șt.N.; 8 specs, 21.07.1979, leg. A.P.-G.; 1 ふ̋, Chilia Veche: 7.05.1964, leg. X.S.-P.; 3 गे, 3 q, Chituc Levee, Sinoe-Black Sea Channel, 17.08.2003, black light traps, leg. C.Ci.; Crișan: 9 specs, 24.06.1967, leg. Șt.N.; 23 specs, 17.08.1967, leg. X.S.-P.; 6 specs, 26.09.1967, leg. Șt.N.; 4 specs, 1.07.1968, leg. Șt.N.; 2 specs, 16.07.1970, leg. Șt.N.; 6 specs, light trap, 18.07.1970, leg. Șt.N.; 3 ò, 1 q, 21.07.1970, leg. Șt.N.; 2 ô, 2 o, 16.07.1970, leg. Șt.N.; Enisala: 3 गे, 3 ㅇ, 14.08.2003, black light trap, leg. C.Ci.; 3 ô, 1 , 16.08.2003, mercury vapor light trap, leg. C.Ci.; Letea Forest: 1 q, 28.07.1962, leg. ?; 1 spec., 19.07.1980, leg. A.P.-G.; 4 ò, 2 ㅇ, Madgearu Channel, 20-21.06.1993, leg. C.H.; 36 specs, Maliuc, Mila 26, 6-9.08.1991, light trap, leg. I.M.\&R.S.; 33 specs, Maliuc, Mila 26, Sulina Channel, 26.06.2002, mercury vapor light trap, leg. C.Ci.; 12 specs, Mila 23, 31.07.2002, white light trap, leg. C.Ci.; 4 त, 6 o, Letea (village), 30.06.2016, black light trap, leg. I.I.; Murighiol: 2 o, 3.08.1979, leg.?; 3 ô, 2 o, Nebunu Lake, 7.07.2015, leg. E.I.; Periprava: 3 ō, 3 ㅇ, 13.07.1964, leg. Șt.N.; 4 specs, 30.04.1965, leg. Șt.N.; 2 ō, 6 q, 30.05.1965, leg. Șt.N.; 1 spec., 4.07.1965, leg. Șt.N.; 1 స̄, 1.08.1965, leg. Șt.N.; 1 spec., 24.06.1966, leg. Șt.N.; 4 specs, 14.07.1966, leg. Șt.N.;

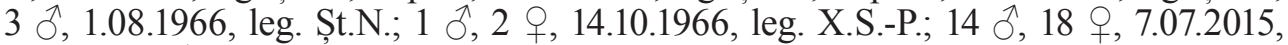
leg. E.I.; 1 đ̃, Periteașca, 17.06.1995, leg. D.R.; Sfântu Gheorghe: 3 specs, 30.06.1971, leg. M.W.; 2 specs, 10.08.1971, leg. ?; 1 spec., 3.07.1971, leg. ?; 1 đo, 1 †, 12.06.1995, leg. D.R.; Sulina: 12 specs, 23.07.1964, leg. Șt.N.; 1 spec., 20.07.1966, leg. Șt.N.; 15 specs, 20-23.07.1966, leg. Șt.N.; 4 specs, 9.07.1967, leg. Șt.N., 4 specs, 7-8.07.1968, leg. A.P.-G.; 3 9, 18.06.1969, leg. Șt.N.; 1 ふૈ, 17.06.1969, leg. I.D.; 2 specs, 18.06.1969, leg. Șt.N.; 1 spec., 11.07.1971, leg. ?; 1 spec., 9.07.1974, leg. A.P.-G.; 5 specs, 1.07.1979, leg. ?; 1 ふे, Uzlina (chalet), 18.06.1991, leg. N.G.

\section{Philonthus quisquiliarius (Gyllenhal, 1810)}

Examined material: Caraorman Forest: 3 ô, 3 ㅇ, 21.09.1965, leg. Șt.N.; 3 specs, 12.05.1968, leg. X.S.-P.; 1 ó, 12.07.1968, leg. M.W.; 1 ○, 13.06.1969, leg. Șt.N.; 1 ō, 16.06.1969, leg. Șt.N.; 3 ō, 4 @, Chituc Levee, Sinoe-Black Sea Channel, 17.08.2003,

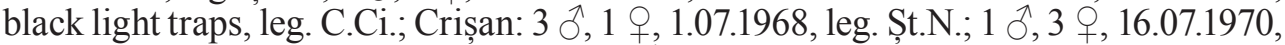
leg. Șt.N.; 2 q, 21.07.1970, leg. Șt.N.; 7 J̃, 6 q, 17.08.1976, leg. X.S.-P.; 21 specs, Enisala, 14.08.2003, black light trap, leg. C.Ci.; 2 9, Letea (village), 30.06.2016, black light trap, leg. I.I.; 1 đ̃, 2 q, Litcov Channel, on the bank, 1.09.1969, leg. Șt.N.;

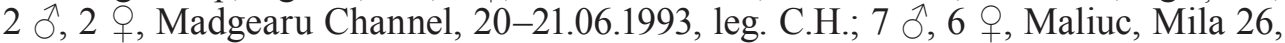
6-9.08.1991, leg. I.M.\&R.S.; 2 ō, 2 q, Maliuc, Mila 26, Sulina Channel, 26.06.2002, mercury vapor light trap, leg. C.Ci.; 4 ग, 1 , Mila 23, 31.07.2002, white light trap, leg. C.Ci; 1 ग̃, 2 ㅇ, Nebunu Lake, 7.07.2015, leg. E.I.; Periprava: 1 spec., 3.07.1963, 
leg. A.P.-G.; 2 specs, 29.07.1963, leg. A.P.-G.; 1 +, Sfântu Gheorghe, 30.06.1971, leg. M.W.; 5 specs, Sulina, 18.06.1969, leg. Șt.N.

\section{Philonthus rectangulus Sharp, 1874}

Examined material: Crișan: 1 + 13.07 .1970 , leg. Șt.N.; 1 ô, 14.07.1970, leg. M.W.; 1, , Maliuc, Mila 26, 6.08.1991, leg. I.M.; 1 o, Madgearu Channel, 20-21.06.1993, leg. C.H.; 1 +, Periprava, 15.10.1966, leg. Șt.N.

\section{Philonthus salinus Kiesenwetter, 1844}

Examined material: 1 गे, C. A. Rosetti, 5.07.1966, leg. Șt.N.; Caraorman Forest: 1 O, 21.06.1967, leg. Șt.N.; 1 đ̂, 23.06.1967, leg. Șt.N.; 1 ô, 13.08.1967, leg. X.S.-P., 7 đ, 5 9, 5.07.1968, leg. St.N.; 2 ô, 1 9, 21.07.1979, leg. A.P.-G.; 6 specs, Chituc Levee, Sinoe-Black Sea Channel, 17.08.2003, black light traps, leg. C.Ci.; Crișan: 1 ô, 18.06.1967, leg. X.S.-P.; 3 ô, 2 o, 17.08.1967, leg. X.S.-P.; 2 ô, 2 क, 1.07.1968, leg. Șt.N.; 1 q, 10.07.1970, leg. Șt.N.; 4 specs, 15.07.1970, leg. Șt.N.; 1 Oे, 21.07.1970, leg. Șt.N.; 3 Oे, 2 + , Enisala, 14.08.2003, black light trap, leg. C.Ci.; 1 q, Hașmacul Mare Natural Reserve, 19.06.1993, leg. I.M.; Letea Forest: 5 ô, 1.04.1966, leg. Șt.N.;

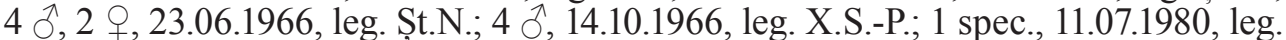
A.P.-G.; 1 Oे, 18.06.1993, leg. I.M.; Letea (village): 3 specs, 4.05.2012, on willow bark

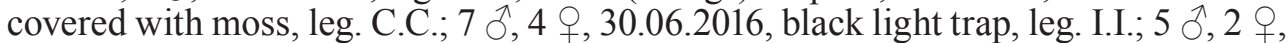
Madgearu Channel, 20-21.06.1993, leg. C.H.; 47 specs, Maliuc, Mila 26, 6-9.08.1991, leg. I.M\&R.S.; 13 specs, Maliuc, Mila 26, Sulina Channel, 26.06.2002, mercury vapor light trap, leg. C.Ci.; 4 specs, Mila 23, 31.07.2002, white light trap, leg. C.Ci.; 1 q, Murighiol, 3.08.1979, leg.?; 6 §,, 3 ㅇ, Nebunu Lake, 7.07.2015, leg. E.I.; Periprava: 2 o, 29.07.1963, leg. A.P.-G.; 1 क, 13.07.1964, leg. S.t.N.; 7 specs, 30.04.1965, leg. Șt.N.;16 specs, 30.05.1965, leg. Șt.N.; 38 specs, 4.07.1965, leg. Șt.N.; 6 ㅇ, 14.10.1966, leg. Șt.N.; 3 ${ }^{2}, 1$ q, Periteașca, 17.06.1995, leg. D.R.; 1 spec., Sarinasuf, 24.06.1962, leg. X.S.-P.; Sulina: 2 ô, 1 ㅇ, 7.06.1963, leg. Șt.N.; 1 ô, 1 ㅇ, 12.07.1967, leg. A.R.; 3 specs, 18.06.1969, leg. Șt.N.; 1 đo, 1 , Sfântu Gheorghe, 12.06.1995, leg. D.R.; 2 specs, Uzlina (chalet), 18.06.1991, leg. N.G.

Philonthus umbratilis (Gravenhorst, 1802)

Examined material: 1 + , Jurilovca, 29.07.1984, leg. N.G.; 3 ภ, 1 ㅇ, Maliuc, Mila 26, 6-9.08.1991, leg. I.M.\&R.S.; 1 +, Nebunu Lake, 7.07.2015, leg. E.I.

Philonthus viridipennis Fauvel, 1875

Examined material: 1 đo, 1 o, Caraorman, 16.06.1969, leg. Șt.N; 1 ô, Maliuc, Mila 26, 6-9.08.1991, leg. R.S.

Philonthus wuesthoffi Bernhauer, 1939

Examined material: 1 ô, Chituc Levee, Sinoe-Black Sea Channel, 17.08.2003, black light trap, leg. C.Ci.; 1 \%, Nebunu Lake, 7.07.2015, leg. E.I.

Staphylinus caesareus (Cederhjelm, 1798)

Examined material: 2 specs, Caraorman Forest, 12.05.1967, leg. M.W.; 1 spec., Periprava, 12.10.1966, leg. X.S.-P.

Staphylinus erythropterus Linnaeus, 1758

Examined material: 1 spec., Periprava, 11.07.1962, leg. M.W. 
Tasgius pedator (Gravenhorst, 1802)

Examined material: 1 गे, Letea (village), 25.09.2013, leg. C.C.

Tasgius winkleri (Bernhauer, 1906)

Examined material: 1 spec., Letea Forest, 9.10.2012, leg. I.I.; 1 + , Periprava, 5.11.1964, leg. N.S.

\section{DISCUSSIONS}

Until now, Bledius spectabilis Kraatz, 1857 was recorded from the Danube Delta. In 2010, M. Schülke revalidated Bledius frisius Lohse, 1978. I have revised the specimens present in Museum's collections under the name B. spectabilis and they belong to the species $B$. frisius. Besides specimens collected from the Danube Delta Biosphere Reserve, there are 23 other specimens of Bledius frisius preserved in the collections recorded from Eșelnița (Mehedinți County) and 1 specimen from Agigea Reserve (Constanța County). $1 \hat{O}$ and $1+$ of Bledius spectabilis Kr. are perserved in Montandon collections of "Grigore Antipa" National Museum of Natural History. On the label is mentioned Bretagne.

In the examined material, two species of Achenium were identified, namely A. propontiacum Bordoni, 2009 and A. humile (Nicolai, 1822). I have revised the Achenium specimens preserved in the Coleoptera collections: 1 ô, 1 \%, Măcin, Dobrudja, coll. Montandon and 1 \%, 3 , , Comana (Giurgiu County), coll. Montandon. These specimens belong to $A$. propontiacum and not to $A$. depressum (Stan, 2006, 2008). In the collections there are $1 \hat{\delta}$ of $A$. humile from Comana, coll. Montandon and 1 from Várhegy (Chinari, Mureș County), coll. Deubel. Three species of Achenium are known from Romania (Assing, 2010). A male of A. depressum (Gravenhorst, 1802) from Pitești, 23.08.1991, leg. Șerban Procheș, is preserved in the Museum collections.

Regarding Ochthephilum species, only $O$. collare (Reitter, 1884), a species with a Ponto-Mediterranean distribution (Assing, 2009), was identified in the Danube Delta Biosphere Reserve. Aside from the Danube Delta specimens, I have revised all the Ochthephilum specimens in the Coleoptera collections and many specimens belong to O. collare: 1 क, Azuga, 29.X.?; 1 क , Poiana Țapului; 3 $\lambda, 1$ \% , Comana, Vlasca (Giurgiu County) coll. Montandon; 2, 4 , Gespreng (Spring, Alba County), coll. Deubel; 1 ô, Izvorul Dulce, Beceni (Buzău County), 4.08.2008, leg. Viorel Ungureanu. In the Museum's collections there are 1 \%, 1 + of $O$. fracticorne (Paykull, 1800) from Crucea (Suceava County, Romania), coll. Montandon; 1 ô, Galatz (Galați), coll. Fleck and 4 , 4 \& from Belarus (Stan \& Khotko, 2011). We have not revised the specimens of Ochthephilum (Cryptobium) cited by Ieniștea (1974) from Caraorman.

\section{ACKNOWLEDGEMENTS}

The present paper is a posthumous homage to the Museum specialists who contributed to the development of the Coleoptera collections: Ion Drăghia, Cristina Hoinic, Ștefan Negru, Aurelian Popescu-Gorj, Atena Roșca, Nicolae Săvulescu, Xenia Scobiola-Palade, Igor Sienkiewicz, Medeea Weinberg. The author expresses her sincere thanks to the colleagues: Rodica Serafim, Ioana Matache, Dr. Ionuț Iorgu, Dr. Elena Iorgu, Dr. Gabriel Chișamera, Dr. Cristina Constantinescu; Dr. Costică Adam, Dr. Angela Petrescu, Dr. Dorel Ruşti, Dr. Nicolae Găldean. Many thanks to Dr. Constantin Ciubuc who donated rove beetle specimens sorted by him from samples collected using light traps. Thanks to Liviu Moscaliuc and Dr. Ionut Iorgu for the photos. Moreover, thanks are extended to my colleague Dr. Alexandru Iftime for generating the map. I also thank the referees for their constructive comments on my manuscript and for their publishing advice. 


\section{REFERENCES}

ASSING, V. (2009) On the Western Palaearctic and Middle Asian species of Ochthephilum Stephens, with notes on Cryptobium koltzei Eppelsheim (Coleoptera: Staphilinidae: Paederinae: Cryptobiina). Linzer biologische Beitráge, 41 (1): 397-426.

ASSING, V. (2010) A revision of Achenium (Coleoptera: Staphylinidae: Paederinae). Nova Supplementa Entomologica (Keltern), 21: 1-190.

IENIŞTEA, M. AL. (1968) Ord. Coleoptera (pars). Travaux du Muséum d'Histoire Naturelle "Grigore Antipa", 9: 97-117. (in French)

IENIŞTEA, M. AL. (1974) Contributions a la connaissance des Coleopteres du Delta du Danube (Le "grind" Caraorman). Travaux du Muséum d'Histoire Naturelle "Grigore Antipa", 14: 239-249. (in French)

NEGRU, ŞT. (1968) Ordinul Coleoptera (Pars). Travaux du Muséum d'Histoire Naturelle "Grigore Antipa", 9: 81-95. (in French)

RAIANU, L. (1963) Contribuţii la cunoaşterea şi răspândirea Familiei Staphylinidae în R. P. Română. Analele Ştiinţifice ale Universităţii “A. I. Cuza” Iaşi, Secţiunea II (Biologie), 9 (1): 81-85. (in Romanian)

SCHÜLKE, M. (2010) Zur Taxonomie und Systematik einiger Arten der Untergattung Bledius Leach 1819 (Coleoptera, Staphylinidae, Oxytelinae). Linzer biologische Beitráge, 42 (2): 1495-1509. (in German)

STAN, M. (2004) Contributions to the faunistic and systematic knowledge of the species of genera Paederidus Mulsant \& Rey, 1878 and Paederus Fabricius, 1775 from Romania (Coleoptera: Staphylinidae: Paederinae). Travaux du Muséum National d'Histoire Naturelle "Grigore Antipa", 47: 151-167.

STAN, M. (2006) Contributions to the knowledge of the rove beetle fauna (Coleoptera: Staphylinidae) of north-west of Dobrogea (Romania). Travaux du Muséum National d'Histoire Naturelle "Grigore Antipa", 49: 185-194.

STAN, M. (2008) New data on the rove betles fauna (Coleoptera: Staphylinidae) from Bucureşti and its surroundings. Travaux du Muséum National d'Histoire Naturelle "Grigore Antipa", 51:369-386.

STAN, M. (2010) On the species of Ocypus Leach of the Carpathian Basin with special reference to the species of Romania (Coleoptera: Staphylinidae: Staphylininae: Staphylinini). Acta Entomologica Serbica, 15 (2): 171-193.

STAN, M. (2012) On the species of Philonthus Stephens (Coleoptera: Staphylinidae: Staphylininae: Staphylinini: Philonthina) in the collections of Romanian natural history museums. Travaux du Muséum National d'Histoire Naturelle "Grigore Antipa", 55 (2) 233 - 276.

STAN, M., L. KHOTKO (2011) Rove beetles (Coleoptera: Staphylinidae) from Berezinsky Biosphere Reserve (Republic of Belarus). In: Protected areas of Belarus, 6: 212-230 (in Russian and English). 